\title{
Quantification of Disease Activity in Chronic Nonbacterial Osteomyelitis by Whole-body Magnetic Resonance Imaging
}

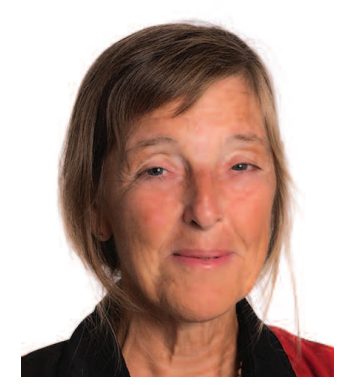

Chronic nonbacterial osteitis (CNO), also termed chronic recurrent multifocal disease (CRMO), is a rare autoinflammatory disease characterized by recurrent flares of inflammatory bone pain related to aseptic osteomyelitis ${ }^{1,2,3}$. It usually affects children and adolescents and the clinical presentations range from mild and limited unifocal disease to severe chronic inflammation in multiple bones ${ }^{4,5,6}$. The long tubular bones of the lower extremities are most commonly involved, followed by the spine and the clavicles $^{2,3}$; there may be extraskeletal skin and bowel manifestations such as palmoplantar pustulosis, psoriasis, and Crohn disease ${ }^{7}$.

Diagnostic imaging in CNO relies on a multimodality approach usually consisting of radiography followed by magnetic resonance imaging (MRI) ${ }^{2}$. Because bone marrow edema (BME) is characteristic for active lesions, fat-saturated fluid sensitive MRI sequences such as short-tau inversion recovery (STIR) and T2-weighted fat-saturated sequence are ideal for detecting active lesions, whereas T1-weighted sequences are preferable for detecting chronic structural lesions ${ }^{2}$. The findings by MRI may, however, be nonspecific and the diagnosis is usually a diagnosis of exclusion after ruling out other diseases, such as infectious osteomyelitis and tumors or tumor-like lesions, based on clinical presentation, imaging, and if needed, culture-negative biopsy $1,7,8$. The combination of characteristic imaging findings are usually diagnostic ${ }^{2,7}$, such as multiple bone lesions or characteristic metaphyseal lesions in the lower extremities, and clavicular or spinal osteitis with concomitant dermal changes.

To diagnose and monitor the extent of $\mathrm{CNO}$ in children/adolescents, a whole-body (WB)-MRI approach is advantageous because radiological disease activity may be underestimated clinically ${ }^{1,5,9}$. WB-MRI gives a simultaneous evaluation of the entire body without exposure to ionizing radiation, and in small children the number of needed MR examinations in sedation can be reduced. Multifocality and clinically silent lesions can be detected by WB-MRI ${ }^{5}$; this is valuable in the decision making regarding treatment and followup because clinical remission does not necessarily mean radiological remission ${ }^{9}$. WB-MRI is therefore increasingly used to assess the total $\mathrm{CNO}$ activity. A valid/reliable scoring system for active as well as chronic lesions detectable by WB-MRI is therefore needed to stage as well as monitor the disease over time. A previous proposal for scoring $\mathrm{CNO}$ in children/adolescents by WB-MRI has mainly been based on the number and size of lesions and their anatomical locations ${ }^{5}$. Another scoring method, predominantly used to evaluate region-specific MRI, was more concentrated on $\mathrm{CNO}$ characteristics ${ }^{10}$. None of these methods has been validated. This may be due to some inherent difficulties in $\mathrm{CNO}$ evaluation related to the variable disease presentations such as a tibio/appendicular multifocal pattern, a claviculo/spinal paucifocal pattern, or mixed presentations ${ }^{6}$. Although the MR appearance of characteristic CNO lesions is well described in areas of predilection, all bones can potentially be involved and the appearance can vary considerably. It is therefore much more difficult to establish a scoring system for $\mathrm{CNO}$ lesions than for the rather homogeneous lesions occurring in axial spondyloarthritis $^{11,12}$ and rheumatoid arthritis ${ }^{13}$.

In this issue of The Journal, Zhao, et al ${ }^{14}$ present a method to score CNO lesions by WB-MRI mainly based on a standardized scoring/grading of the different characteristics of CNO lesions, irrespective of the bone involved. This chronic nonbacterial osteomyelitis MRI scoring (CROMRIS) system is based on CNO features previously used to score region-specific $\mathrm{MRI}^{10}$, but with modifications developed in consensus by 11 pediatric radiologists from 7 different hospitals in North America and Europe. The following 4 $\mathrm{CNO}$ characteristics applicable for all CNO lesions were defined to be scored as shown in Table 1: bone marrow hyperintensity, signal size, soft tissue or periosteal hyperintensity, and bony expansion. The scoring of these features was supported by an atlas, encompassing representative MRI of active $\mathrm{CNO}$ lesions.

In addition, the following $\mathrm{CNO}$ manifestations were defined to be scored (Table 1): vertebral compression, kyphosis, limb hypertrophy, and joint effusion, and the

See MRI scoring for CNO, page 739 
Table 1. Variables in the chronic nonbacterial osteomyelitis MRI scoring (CROMRIS) system ${ }^{14}$.

\begin{tabular}{|c|c|}
\hline \multicolumn{2}{|c|}{$\mathrm{CNO}$ characteristics applicable for all $\mathrm{CNO}$ lesions } \\
\hline Variables & Score values \\
\hline Bone marrow hyperintensity & Absent,$<$ fluid signal, and similar to fluid signal ${ }^{\#}$ \\
\hline Signal size & Absent, $<25 \%, 25-50 \%$, and $>50 \%$ of the bone involved \\
\hline Soft tissue or periosteal hyperintensity & Absent or present \\
\hline Bony expansion & Absent or present \\
\hline \multicolumn{2}{|l|}{ Region-specific CNO features } \\
\hline Variables of interest & Score values \\
\hline Vertebral compression & Normal, some height loss, and vertebra plana \\
\hline Kyphosis & Absent or present \\
\hline Limb hypertrophy & Absent or present \\
\hline Joint effusion & Absent or present \\
\hline \multicolumn{2}{|l|}{ Grouping of bones } \\
\hline Complex bones & $\begin{array}{l}\text { Scapula, sacrum (combine } \mathrm{S} 1-5 \text { for each side), pelvis (ilium, } \\
\text { periacetabulum, pubis/ischium), ribs, mandible, skull, patella, } \\
\text { clavicle, divided into laterality (right and left side), and sternum } \\
\text { (manubrium and sternal body) }\end{array}$ \\
\hline Long bones & $\begin{array}{l}\text { Humerus, ulna, radius, femur, tibia, fibula. These bones were } \\
\text { divided anatomically into proximal epiphysis, proximal } \\
\text { metaphysis, diaphysis, distal metaphysis, and distal epiphysis }\end{array}$ \\
\hline Less-commonly affected bones & $\begin{array}{l}\text { Hand: carpals, metacarpals, and phalanges; hindfoot: talus and } \\
\text { calcaneus; fore/mid-foot: tarsals, metatarsals, phalanges }\end{array}$ \\
\hline Spine & $\begin{array}{l}\text { Sum of individual vertebral bodies from cervical to lumbar region } \\
\text { with concomitant dichotomous scoring of abnormal signals } \\
\text { within lateral and posterior spinal structures. }\end{array}$ \\
\hline
\end{tabular}

\# Additional scoring of confidence level as low, medium, or high. CNO: chronic nonbacterial osteitis; MRI: magnetic resonance imaging.

bones were grouped into complex bones, long bones, less-commonly affected bones, and the spine.

The interrater agreement and reliability of the scoring system was tested by 11 readers based on 45 WB-MRI; 41 patients with $\mathrm{CNO}$ and 4 with other diseases. The results regarding the presence or absence of BME in the different regions are not given, but it is stated that there is a low absolute agreement of signal hyperintensity in the commonly affected bones (tibia and femur). Therefore, a predefined 70\% agreement was used as a threshold to determine whether there was a "true" abnormal bone marrow signal. The absolute interrater agreement of the signal intensity of BME was low $(<60 \%)$ in more commonly affected bones such as the femur, tibia, fore/mid-foot, hind foot, and clavicle. Most of the low- and medium-confidence readings were from commonly affected sites such as femur, tibia, and foot, whereas the absolute agreement was near or greater than $80 \%$ in the majority of less-commonly affected bones. The agreement regarding the size of BME was in most segments of the femur and tibia lower than in other long bones. These relatively lower agreements regarding the most commonly involved sites indicate a need for further calibration regarding the detection of BME areas and scoring of their features for intensity and size. It is important to have a valid detection and grading of lesions in commonly affected bones, if the scoring system is used to monitor the disease over time, including to monitor the effect of therapy.
The authors are, however, to be congratulated on their effort and the resulting cross-sectional validation analysis, which is an important step toward a common scoring system in $\mathrm{CNO}$.

The scoring system it a potential research tool, but may be too complicated to be used in clinical practice, where the standardized reporting of WB-MRI findings proposed by Arnoldi, et $a l^{5}$ may be more feasible. Their radiologic index for nonbacterial osteitis (RINBO) includes the absolute size of active lesions and clustering the number of active lesions into 3 categories: unifocal, paucifocal (2-4 lesions), and multifocal (5 or more lesions; Table 2). Soft tissue inflammation, periosteal reaction, and hyperostosis were classified as extramedullary findings, and spinal involvement was divided into active with abnormal STIR signal and chronic lesions with deformation (Table 2$)^{5}$. The use of a relative lesion size in CROMRIS is more appropriate for the pediatric population, indicating a need for modifications of the RINBO when used. Although less detailed, RINBO gives the possibility of estimating a total score of up to 10 . This is not yet a feature of CROMRIS, because further studies were deemed necessary to determine the weight of each variable. A score taking all lesions into account is needed because the fluctuating course implies that new lesions may occur while others are vanishing ${ }^{4,9}$.

Future studies within WB-MRI scoring methods in CNO should be focused on the following aspects:

Personal non-commercial use only. The Journal of Rheumatology Copyright (C) 2020. All rights reserved. 
Table 2. The radiologic index for WB-MRI in patients with $\mathrm{CNO}$ and RINBO 5 .

\begin{tabular}{lcc}
\hline Variables of Interest & $\begin{array}{c}\text { RINBO } \\
\text { Criterion }\end{array}$ & Value \\
\hline $\begin{array}{l}\text { No. of patient's radiologically active lesions (RAL) } \\
\text { Unifocal }\end{array}$ & 1 & 1 \\
Paucifocal & $2-4$ & 2 \\
Multifocal & $\geq 5$ & 3 \\
Maximum size of patient's RAL & $<10 \mathrm{~mm}$ & 1 \\
Minor & $10-100 \mathrm{~mm}$ & 2 \\
Average & $>1000 \mathrm{~mm}$ & 3 \\
Major & & \\
Extramedullary involvement & Periostal reaction and/or soft tissue edema & 1 \\
Acute & & 1 \\
Chronic & Hyperostosis & 1 \\
Spinal involvement & Radiologically active vertebral lesion & 1 \\
Acute & CNO-related vertebral body deformation & \\
Chronic & & 10 \\
Maximum index point & & \\
\hline
\end{tabular}

WB-MRI: whole-body magnetic resonance imaging; CNO: chronic nonbacterial osteitis; RINBO: radiologic index for nonbacterial osteitis.

(1) Validity and sensitivity to changes of CNO activity by WB-MRI in longitudinal studies

(2) Elaboration of a total score for radiological disease activity on a whole-body level

(3) Standardization of the WB-MRI protocol between pediatric centers.

There is currently no consensus among centers regarding protocols for pediatric WB-MRI, leading to divergent approaches and limiting comparison among centers ${ }^{15}$. Standardizing WB-MRI protocols will ease the implementation of uniform scoring. In this context, it is important to remember that a coronal STIR sequence can be regarded as an inverted bone scan, and may not alone be sufficient for CNO evaluation. A T1-weighted sequence will provide further information about the bone marrow, which often is needed to handle the major challenge defining thresholds for normality versus pathology in children taking the gradual conversion of the bone marrow into account ${ }^{16}$.

A protocol consisting of coronal body slices in addition to sagittal imaging of the spine may have a low sensitivity regarding some anatomical structures such as the ribs and sternum ${ }^{1}$. This can probably be overcome by acquiring a 3-D STIR sequence with axial reconstructions ${ }^{17}$ or performing an axial WB sequence ${ }^{18}$. In accordance with that practice, axial STIR or T2 fat-saturated sequence was obtained in over half of pediatric patients in North America, whereas coronal T1 was performed in about half of the patients ${ }^{15}$.

A universal problem with WB-MRI is that the upper extremities may not be included in the scan field in large children. In an analysis by Arnoldi, et al, restricted coverage of the upper extremity occurred in $58 \%(\mathrm{n}=23)$ of the patients ( 15 elbow and 16 distal radius) ${ }^{5}$. It may therefore be necessary to perform additional sequences of the arms ${ }^{1}$.
Functional MR techniques such as diffusion-weighted MRI (DWI), which is widely used in oncology ${ }^{16}$, should be kept in mind. Although its reliability has not been assessed in patients with $\mathrm{CNO}$, it has been shown that the DWI-apparent diffusion coefficient (ADC) values are significantly higher in lesions compared to normal reference tissue $^{19}$, and DWI can be used to differentiate CNO lesions from tumors with lower ADC values ${ }^{19}$.

\author{
ANNE GRETHE JURIK (1), MD, DMSc, \\ Consultant and Professor, \\ Department of Radiology, \\ Aarhus University Hospital, \\ Aarhus, Denmark
}

Address correspondence to Dr. A.G. Jurik, Department of Radiology, Aarhus University Hospital, Palle Juul-Jensens Boulevard 35, Entrance C, location C118/ reference point C109, 8200 Aarhus N, Denmark.

E-mail: annejuri@rm.dk

\section{REFERENCES}

1. Damasio MB, Magnaguagno F, Stagnaro G. Whole-body MRI: n on-oncological applications in paediatrics. Radiol Med 2016; 121:454-61.

2. Jurik AG, Klicman RF, Simoni P, Robinson P, Teh J. SAPHO and CRMO: the value of imaging. Semin Musculoskelet Radiol 2018;22:207-24.

3. Zhao Y, Ferguson PJ. Chronic nonbacterial osteomyelitis and chronic recurrent multifocal osteomyelitis in children. Pediatr Clin North Am 2018;65:783-800.

4. Andreasen CM, Jurik AG, Glerup MB, Host C, Mahler BT, Hauge $\mathrm{EM}$, et al. Response to early-onset pamidronate treatment in chronic nonbacterial osteomyelitis: a retrospective single-center study. J Rheumatol 2019;46:1515-23,

5. Arnoldi AP, Schlett CL, Douis H, Geyer LL, Voit AM, Bleisteiner F, et al. Whole-body MRI in patients with non-bacterial osteitis: radiological findings and correlation with clinical data. Eur Radiol 2017;27:2391-9.

6. Andronikou S, Mendes da Costa T, Hussien M, Ramanan AV. Radiological diagnosis of chronic recurrent multifocal osteomyelitis using whole-body MRI-based lesion distribution patterns. Clin Radiol 2019;74:737.e3,737.e15.

7. Jansson A, Renner ED, Ramser J, Mayer A, Haban M, Meindl A, et al. Classification of non-bacterial osteitis: retrospective study of clinical, immunological and genetic aspects in 89 patients. Rheumatology 2007;46:154-60.

8. Jurik AG, Egund N. MRI in chronic recurrent multifocal osteomyelitis. Skeletal Radiol 1997;26:230-8.

9. Voit AM, Arnoldi AP, Douis H, Bleisteiner F, Jansson MK, Reiser $\mathrm{MF}$, et al. Whole-body magnetic resonance imaging in chronic recurrent multifocal osteomyelitis: clinical longterm assessment may underestimate activity. J Rheumatol 2015;42:1455-62.

10. Zhao Y, Chauvin NA, Jaramillo D, Burnham JM. Aggressive therapy reduces disease activity without skeletal damage progression in chronic nonbacterial osteomyelitis. J Rheumatol 2015;42:1245-51.

11. Maksymowych WP, Inman RD, Salonen D, Dhillon SS, Krishnananthan R, Stone M, et al. Spondyloarthritis Research Consortium of Canada magnetic resonance imaging index for assessment of spinal inflammation in ankylosing spondylitis. Arthritis Rheum 2005;53:502-9.

12. Maksymowych WP, Inman RD, Salonen D, Dhillon SS, Williams M, Stone M, et al. Spondyloarthritis Research Consortium of 
Canada magnetic resonance imaging index for assessment of sacroiliac joint inflammation in ankylosing spondylitis. Arthritis Rheum 2005;53:703-9.

13. Ostergaard M, Peterfy CG, Bird P, Gandjbakhch F, Glinatsi D, Eshed I, et al. The OMERACT Rheumatoid Arthritis Magnetic Resonance Imaging (MRI) Scoring System: updated recommendations by the OMERACT MRI in Arthritis Working Group. J Rheumatol 2017;44:1706-12.

14. Zhao Y, Sato TS, Nielsen SM, Beer M, Huang M, Iyer RS, et al. Development of a scoring tool for chronic nonbacterial osteomyelitis magnetic resonance imaging and evaluation of its interrater reliability. J Rheumatol 2020;47:739-47.

15. Schooler GR, Davis JT, Daldrup-Link HE, Frush DP. Current utilization and procedural practices in pediatric whole-body MRI. Pediatr Radiol 2018;48:1101-7.
16. Nievelstein RA, Littooij AS. Whole-body MRI in paediatric oncology. Radiol Med 2016;121:442-53.

17. Merlini L, Carpentier M, Ferrey S, Anooshiravani M, Poletti PA, Hanquinet S. Whole-body MRI in children: Would a 3D STIR sequence alone be sufficient for investigating common paediatric conditions? A comparative study. Eur J Radiol 2017;88:155-62.

18. Jurik AG, Jørgensen PH, Mortensen MM. Whole-body MRI in assessing malignant transformation in multiple hereditary exostoses and enchondromatosis: audit results and literature review. Skeletal Radiol 2019;49:115-24.

19. Leclair N, Thormer G, Sorge I, Ritter L, Schuster V, Hirsch FW. Whole-body diffusion-weighted imaging in chronic recurrent multifocal osteomyelitis in children. PLoS One 2016;11:e0147523.

J Rheumatol 2020;47:646-9; doi:10.3899/jrheum.191136 